\title{
Performance of the $x$-ray free-electron laser oscillator with crystal cavity
}

\author{
R. R. Lindberg, ${ }^{1, *}$ K.-J. Kim, ${ }^{1}$ Yu. Shvyd'ko, ${ }^{1}$ and W. M. Fawley ${ }^{2}$ \\ ${ }^{1}$ Advanced Photon Source, Argonne National Laboratory, Argonne, Illinois 60439, USA \\ ${ }^{2}$ Center for Beam Physics, Lawrence Berkeley National Laboratory, Berkeley, California 94720, USA
}

(Received 19 July 2010; published 28 January 2011)

\begin{abstract}
Simulations of the x-ray free-electron laser (FEL) oscillator are presented that include the frequencydependent Bragg crystal reflectivity and the transverse diffraction and focusing using the two-dimensional FEL code GINGER. A review of the physics of Bragg crystal reflectors and the x-ray FEL oscillator is made, followed by a discussion of its numerical implementation in GINGER. The simulation results for a two-crystal cavity and realistic FEL parameters indicate $\sim 10^{9}$ photons in a nearly Fourier-limited, ps pulse. Compressing the electron beam to $100 \mathrm{~A}$ and $100 \mathrm{fs}$ results in comparable $\mathrm{x}$-ray characteristics for relaxed beam emittance, energy spread, and/or undulator parameters, albeit in a larger radiation bandwidth. Finally, preliminary simulation results indicate that the four-crystal FEL cavity can be tuned in energy over a range of a few percent.
\end{abstract}

DOI: 10.1103/PhysRevSTAB.14.010701

PACS numbers: 41.60.Cr

\section{INTRODUCTION}

With the success of the Linear Coherent Light Source (LCLS) [1], we are entering the era of x-ray free-electron lasers (FELs) that realize an enormous improvement in brightness and coherence over that possible with thirdgeneration synchrotron x-ray sources. Similar devices that are also based on self-amplified spontaneous emission (SASE) are under construction in Japan [2] and Europe [3], while several others have been proposed. Typical characteristics of SASE pulses include high transverse coherence, short temporal durations $\lesssim 100 \mathrm{fs}$, high peak intensity, and photon number per pulse $\left(\sim 10^{12}\right)$, but a temporally chaotic structure whose normalized spectral bandwidth is $\sim 0.1 \%$.

Recently, an x-ray FEL oscillator [4] was proposed in the 5-20 keV energy range that can potentially offer complementary performance to sources based on SASE, with $\sim 10^{3}$ lower peak powers, $\sim 10^{3}$ narrower spectral bandwidth, and $\sim 10^{3}$ higher repetition rate. The peak spectral brightness is comparable to a SASE device, while the repetition rate and the corresponding average spectral brightness is increased by $\sim 10^{3}$. An FEL oscillator cavity can be made for $\mathrm{x}$ rays using near-normal-incidence Bragg mirrors to trap the radiation (as was first recognized by Colella and Luccio [5]), and grazing incidence mirrors to provide the $\mathrm{x}$-ray focusing.

Designing an X-ray FEL oscillator requires the synthesis of several components operating near the present edge of technological capabilities, including the production and acceleration of high-brightness electron beams, the optimization of radiation generation in the undulator, and the reflection and manipulation of the $\mathrm{x}$-ray pulses so as to overlap the subsequent electron bunch and yield FEL gain.

\footnotetext{
*lindberg@aps.anl.gov
}

This paper begins to address the latter two areas, i.e., the physics of the x-ray FEL cavity, by incorporating Bragg diffraction of $\mathrm{x}$ rays from crystals into the well-known axisymmetric FEL code GINGER [6]. In this manner, various FEL parameter and design studies can be done that model both the relevant electron beam physics (such as energy spread, emittance, and longitudinal current profile) and the radiation propagation (including reflection, filtering, and focusing by crystals and mirrors).

We devote the following two sections to discussing the physics relevant to our x-ray FEL oscillator simulations. We first review dynamical Bragg diffraction theory of $\mathrm{x}$ rays from crystals in Sec. II, showing that the reflected radiation from a crystal will be attenuated by a few percent, filtered in frequency, and delayed in time. Consideration of the FEL oscillator physics in Sec. III demonstrates that these crystal properties put certain requirements on the single-pass FEL gain and electron beam duration, while also implying that the oscillator cavity length must be slightly shortened from normal synchronism to ensure appropriate electron beam-radiation overlap. Additionally, we discuss incorporating arbitrary Bragg crystal reflectivities (which are typically calculated theoretically, but could be determined experimentally) into the 2D FEL code GINGER. The present work takes advantage of the decoupling between transverse and temporal dimensions that occurs for Bragg reflections near backscattering; future work will include the angular dependence of the crystal reflectivity. Finally, in Sec. IV we use the modified GINGER code to perform a number of simulations of the x-ray FEL oscillator including the spectral reflectivity of the Bragg crystals and transverse diffraction and focusing. Using a low-charge $(\sim 25-50 \mathrm{pC})$, low normalized emittance $\left(\varepsilon_{x} \sim 0.2 \mathrm{~mm} \mathrm{mrad}\right)$ electron beam in near direct backscattering, we show that tens of MW of longitudinally coherent radiation in the energy range from 5 to $20 \mathrm{keV}$ can be 
produced in a $\sim 1$ ps pulse. Additionally, we show that by compressing the low-charge beam by a factor of 10 (i.e., to $100 \mathrm{fs}$ width and peak current of $100 \mathrm{~A}$ ), we can relax the requirements on total cavity reflectivity, beam parameters, and/or undulator length, while still achieving MWs of coherent output power. Finally, via a four-mirror geometry, preliminary simulations show that tens of MW of $14 \mathrm{keV}$ $\mathrm{x}$ rays can be produced and varied over a photon energy range $\sim 6 \%$.

\section{BRAGG CRYSTAL MIRRORS}

In this section, we briefly review those results of the dynamical theory of $\mathrm{x}$-ray Bragg diffraction from crystals that are most relevant to understanding the physics of $\mathrm{x}$-ray FEL oscillator Bragg crystal mirrors. We adopt the notation of Ref. [7], quoting the main formulas detailing the crystal response, while subsequent discussion focuses on how the crystal properties affect FEL performance.

We consider a Bragg reflector whose reflecting atomic planes are oriented at an angle $\eta$ (the asymmetry angle) from the crystal surface as shown in Fig. 1(a); symmetric scattering is defined by $\eta=0$. The incident $\mathrm{x}$ rays make an angle $\Theta$ from the crystal plane normal, and have a wavelength $\lambda$ and energy $E=h c / \lambda$ (where $h$ is Planck's constant and $c$ is the speed of light). Coherent reflection from the mirror surface occurs when the wavelength of the incident radiation nearly satisfies Bragg's law for reflection: $\lambda=2 d_{H} \cos \Theta \equiv \lambda_{H} \cos \Theta$, where $d_{H}$ is the spacing between crystal planes, which in turn defines the Bragg wavelength via $\lambda_{H}=2 d_{H}$. We schematically represent the band of large coherent Bragg reflectivity when $\lambda \approx$ $\lambda_{H} \cos \Theta$ in Fig. 1(b). Note how near backscattering, i.e., for $\Theta \ll 1$, the shaded region of high reflectivity is nearly
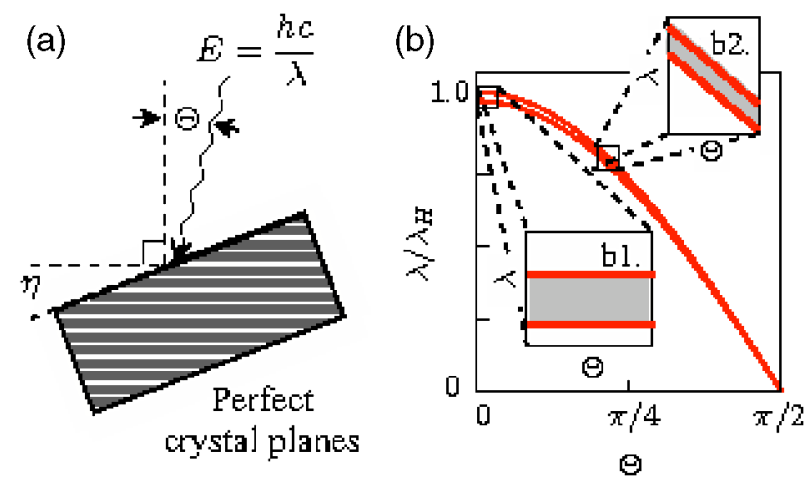

FIG. 1. (a) General Bragg diffraction geometry of $x$ rays from a crystal. The incident radiation makes an angle $\Theta$ from the normal to the reflecting atomic planes, which differs from the surface normal by the asymmetry angle $\eta$. (b) Bragg diffraction occurs in a small band in $\lambda$ and $\Theta$ near the Bragg condition $\lambda=$ $\lambda_{H} \cos \Theta$. Near backscattering $(\Theta \ll 1)$, the reflectivity is nearly independent of $\Theta$ as shown by the shaded area in box (b1). For generic incidence angles, the reflectivity range is given by the box (b2).
$\Theta$ independent as depicted in box (b1), while at a generic angle the local reflectivity can be approximated by a band in the $\Theta-\lambda$ plane whose slope $\approx-\lambda_{H} \sin \Theta$ as indicated by the box (b2) in Fig. 1(b).

The interaction between the incident radiation and the crystal medium is given by the electric susceptibility $\chi(\boldsymbol{r})$, which can be expanded in a Fourier series by virtue of the crystalline periodicity. Denoting the reciprocal lattice vectors of the crystal by $\boldsymbol{H}_{n}$, we have

$$
\chi(\boldsymbol{r})=\sum_{H_{n}} \chi_{H_{n}} e^{i \boldsymbol{H}_{n} \cdot \boldsymbol{r}} .
$$

We assume that the crystal and its orientation have been chosen to support a single Bragg reflection, meaning that the resonant excitation condition is only satisfied for the two waves whose reciprocal lattice vectors are $\boldsymbol{H}_{0}=0$ and $\pm \boldsymbol{H}_{1}$ (in the $\mathrm{x}$-ray optics literature this is referred to as two-beam Bragg diffraction). To simplify notation, we denote the corresponding Fourier coefficients as $\chi_{0}, \chi_{H}$, and $\chi_{\bar{H}}$, respectively. In the general case $\chi_{H}$ are complex; however, for a nonabsorbing, centrosymmetric crystal lattice we have $\chi_{H}=\chi_{\bar{H}}$. To account for the narrow bandwidth of strongly reflected radiation, we introduce the parameter of deviation from the Bragg reflection condition $\alpha$, which can be written as [7]

$$
\alpha=\frac{4 \lambda}{\lambda_{H}}\left(\frac{\lambda}{\lambda_{H}}+\cos \Theta\right)=\frac{4 E_{H}}{E}\left(\frac{E_{H}}{E}+\cos \Theta\right),
$$

where $E_{H} \equiv h c / \lambda_{H}$ is the Bragg energy. Using the dynamical theory of Bragg diffraction, the complex amplitude reflection and transmission coefficients can be determined as a function of the deviation parameter $\alpha$; we merely quote the results here. The complex reflection coefficient is given by

$$
r=R_{1} R_{2} \frac{1-e^{i d\left(\varkappa_{1}-x_{2}\right)}}{R_{2}-R_{1} e^{i d\left(\varkappa_{1}-\varkappa_{2}\right)}},
$$

while the transmission coefficient is

$$
t=e^{i \varkappa_{1} d} \frac{R_{2}-R_{1}}{R_{2}-R_{1} e^{i D\left(x_{1}-\varkappa_{2}\right)}}
$$

where $d$ is the crystal thickness, while $R_{1,2}$ and $\varkappa_{1,2}$ depend on the deviation parameter $\alpha$, the Fourier coefficients of the susceptibility $\chi$, and the reflection geometry. At present, it will suffice to give $R_{1,2}$ and $\chi_{1,2}$ in symmetric scattering $(\eta=0)$ for the polarization component parallel to the crystal surface:

$$
\begin{gathered}
R_{1,2} \equiv \frac{1}{2 \chi_{\bar{H}}}\left\{\left(\alpha-2 \chi_{0}\right) \pm \sqrt{\left(\alpha-2 \chi_{0}\right)^{2}-\chi_{H} \chi_{\bar{H}}}\right\} \\
\chi_{1,2} \equiv \frac{\pi}{\lambda \cos \Theta}\left(\chi_{\bar{H}} R_{1,2}+\chi_{0}\right) .
\end{gathered}
$$

Note that the polarization component chosen requires that we orient the planar undulator such that the electric field 
vector is perpendicular to the page of Figs. 1 and 4 ( $\sigma$ polarization); choosing the orthogonal electric field polarization (in the page $/ \pi$ polarization) results in a narrower spectral and angular acceptance of the crystal by a factor $\sim \cos (2 \Theta)$ with no reduction in the peak reflectivity.

We present the basic characteristics of the complex reflectivity curve in the next subsection by analyzing $r(\alpha)$ for $\Theta \ll 1$ assuming a semi-infinite crystal that has no photoabsorption. Although idealized, this is a good first approximation to crystals such as diamond that will yield valuable insight into the reflection physics from a crystal mirror. We then briefly discuss the effects of absorption and finite crystal thickness, while saving the generalization to $\Theta \sim O(1)$ and $\eta \neq 0$ for our discussion of the tunable, four-mirror geometry in Sec. IV C. In this case, the narrow angular acceptance of the Bragg crystal will, in general, require a radial Hankel transform of the electric field; we discuss this extension further in Sec. IIID.

\section{A. Backscattering from an idealized symmetric crystal that is semi-infinite and absorption free}

To understand the essential properties of Bragg crystal mirrors, we first simplify the reflection coefficient (3) by assuming that the crystal is semi-infinite, has no absorptive losses, and the reflecting atomic planes are parallel to the surface $(\eta=0)$. In this case, $e^{i d \chi_{1}} \rightarrow 0$ and the Fourier coefficients $\chi_{0}$ and $\chi_{H}$ are purely real. Furthermore, we choose a near-backscattering geometry where $\Theta \ll 1$, $\alpha \approx-4\left(E-E_{H}\right) / E_{H}$, and define

$$
y \equiv-\frac{\alpha+2 \chi_{0}}{2\left|\chi_{H}\right|} \approx \frac{1}{\left|\chi_{H}\right|}\left[\frac{2\left(E-E_{H}\right)}{E_{H}}+\chi_{0}\right] .
$$

By choosing the appropriate branch of the square root, the reflection coefficient (3) can be written in the following simple form:

$$
r(y)= \begin{cases}y-\sqrt{y^{2}-1} & \text { if } y \geq 1 \\ y-i \sqrt{1-y^{2}} & \text { if }|y| \leq 1 \\ y+\sqrt{y^{2}-1} & \text { if } y \leq-1 .\end{cases}
$$

Writing $r \equiv|r| e^{i \phi}$, we plot the intensity reflectivity $|r|^{2}$ as a green dashed line and the complex phase $\phi$ using a dotted blue line in Fig. 2. This graph indicates that the crystal reflectivity is unity for $|y| \leq 1$, which defines the crystal Darwin width, while dropping rapidly outside of this window. Using the definitions ( 7 ) and ( 2 ) for $\Theta \ll 1$, the peak reflectivity is centered at an energy slightly different from the Bragg energy,

$$
E_{c}=E_{H}\left(1-\chi_{0} / 2\right) \sim E_{H}\left[1+O\left(10^{-4} \text { to } 10^{-6}\right)\right],
$$

while the normalized bandpass/Darwin width of the reflectivity curve is

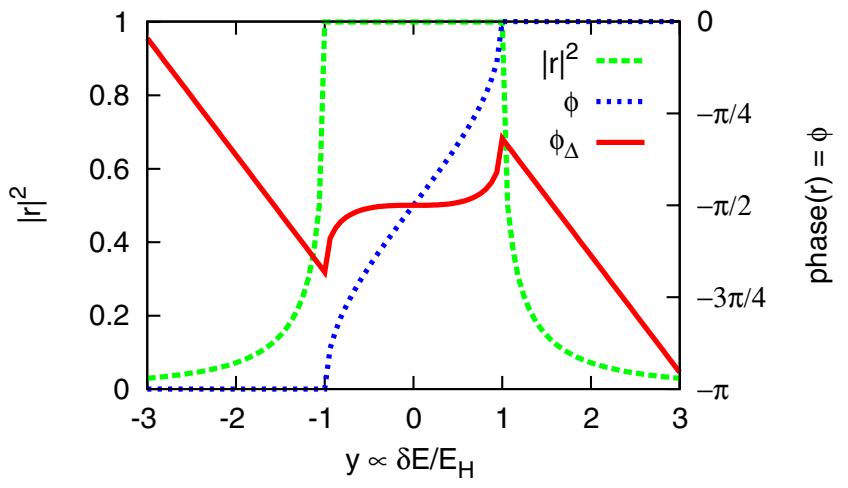

FIG. 2. Reflectivity curve for a semi-infinite, absorption-free crystal. The idealized intensity reflectivity $|r|^{2}$ is the dashed (green) line, showing the Darwin width of peak reflectivity for $|y| \leq 1$, and the sharp decrease outside this window. The complex phase of $r$ (blue dotted line) varies such that $-\pi \leq \phi \leq 0$ within this window, and the mirror acts as a dispersive element. To compensate for the linear part of the dispersion, we slightly decrease the cavity length, and plot the complex phase $\phi_{\Delta}$ of the effective reflection plus cavity length reduction given in (12) as the solid red line. About $y=0$, the phase $\phi_{\Delta}$ is nearly constant, so that the radiation delay has been compensated and the reflected pulse is minimally distorted.

$$
\left.\frac{\Delta E}{E_{H}}\right|_{|r|^{2}=1}=4\left|\chi_{H}\right| \sim 10^{-4} \text { to } 10^{-8} .
$$

Besides the narrow bandwidth of the reflectivity curve, (8) and Fig. 2 also indicate that the crystal imposes a frequency-dependent phase shift on the incident radiation. Near the central wavelength where $|y| \lesssim 1$, the phase shift $\phi$ is a nearly linear function of wavelength, which serves to predominantly delay the incident pulse in time. Expanding (8) for $|y| \ll 1$, the reflectivity is

$$
r(y) \approx-i\left[1+i y-\frac{1}{2} y^{2}+O\left(y^{3}\right)\right] \approx-i e^{i y} .
$$

The factor of $-i$ accounts for the specular reflection phase shift of $-\pi / 2$, while the part dependent on $y$ predominantly [i.e., to $O\left(y^{3}\right)$ ] gives rise to a delay in the radiation. To explicitly demonstrate this, we recall that $y$ is linearly related to the deviation parameter, and write $y=\omega \tau+y_{0}$ for $\tau=2 \hbar / E_{H}\left|\chi_{H}\right|, y_{0}=\chi_{0} /\left|\chi_{H}\right|$, and $\hbar=h / 2 \pi$. In this case, the action of the Bragg crystal in the region of high reflectivity on the radiation electric field $\mathcal{E}(t)$ is approximately given by

$$
\mathcal{E}(t) \rightarrow-\frac{i e^{i y_{0}}}{2 \pi} \int d \omega e^{-i \omega(t-\tau)} \tilde{\mathcal{E}}(\omega) \propto \mathcal{E}(t-\tau),
$$

where $\tilde{\mathcal{E}}$ is the Fourier transform of $\mathcal{E}$. Thus, the radiation observed at time $t$ after Bragg reflection is that which was incident on the mirror at time $t-\tau$ (modulo a constant phase), so that near the central wavelength the mirror predominantly delays the radiation in time [through $O\left(|y|^{2}\right)$ ]. Because the Bragg crystal mirror operates via the coherent superposition of radiation reflected from 
many layers of the crystal, this effective delay can be attributed to the time required for the radiation to penetrate the layers of the lattice; this penetration distance $c \tau=2 h c / E_{H}\left|\chi_{H}\right|$ is twice the crystal extinction length, defined to be the distance at which the field intensity decreases by $1 / e$.

Because the FEL gain requires precise timing between the radiation and electron bunches on each successive pass, the delay in the radiation due to mirror reflection must be compensated by slightly decreasing the optical cavity length. For each mirror, the optimal adjustment decreases the cavity length by an amount $\Delta z=c \tau / 2$, given by

$$
\Delta z=\frac{\lambda_{H}}{2 \pi\left|\chi_{H}\right|} .
$$

Typically, $\Delta z$ is of order $10^{3}$ to $10^{5}$ radiation wavelengths, or $\sim 1-100 \mu \mathrm{m}$. To illustrate how both the reflection and detuning impact the incident radiation, we combine these two elements via the effective reflectivity $r_{\Delta}$. We multiply the reflectivity (8) by the ideal phase shift $e^{-i y}$ associated with the length decrease in (11), and obtain the following effective reflection coefficient:

$$
r_{\Delta}(y)=e^{-i y} \begin{cases}y-\sqrt{y^{2}-1} & \text { if } y \geq 1 \\ y-i \sqrt{1-y^{2}} & \text { if }|y| \leq 1 \\ y+\sqrt{y^{2}-1} & \text { if } y \leq-1 .\end{cases}
$$

We plot the complex phase $\phi_{\Delta}$ of $r_{\Delta}(y)$ as the red solid line in Fig. 2. Note how the cavity length reduction has compensated for the linear increase in $\phi$ within the Darwin width, so that the complex phase is now nearly constant for $|y| \lesssim 1 / 2$. As we will see, the spectrum of the amplifying radiation is typically sufficiently narrow so as to overlap only with this nearly constant in phase region of the reflectivity cure. In this case, the pulse will remain appropriately timed so as to overlap with the next electron beam, while experiencing minimal distortion. In the following subsection we qualitatively discuss the effect of finite thickness and nonzero absorption in the crystal reflectivity, and discuss how these parameters impact FEL performance.

\section{B. Real crystals: Reflection and transmission including absorption and finite thickness}

In the previous subsection, we detailed the basic reflective properties of an idealized symmetric crystal that is semi-infinite and absorption free. Real Bragg crystals suitable for an x-ray FEL oscillator differ in two important respects: the Fourier coefficients of $\chi$ are complex, and the thickness of the crystal is finite. The former manifests itself in photoabsorption that reduces the peak reflectivity and puts a thermal load on the mirror. Finite crystal thickness gives rise to oscillations in $R$ seen in Fig. 3 and can lead to nonzero transmission, which in turn can be exploited by tailoring the mirror thickness to couple some fraction of the radiation out of the cavity.
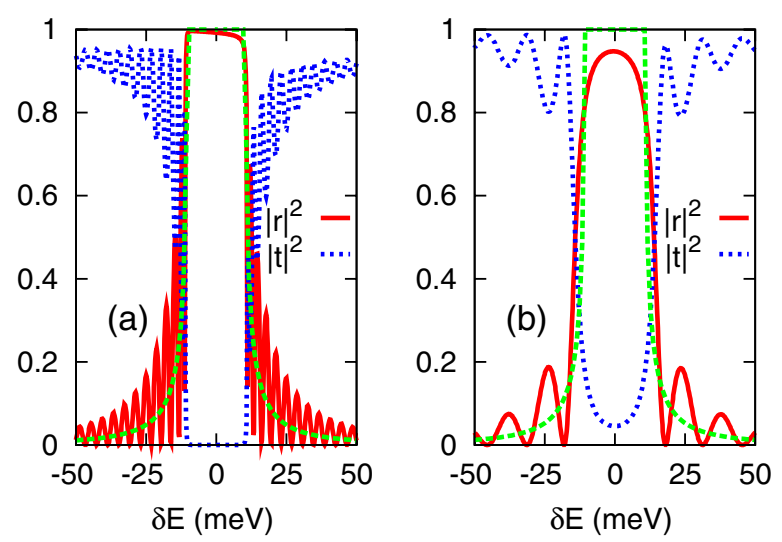

FIG. 3. Intensity reflectivity and transmittance for the diamond crystal C(4 4 4) in exact backscattering. The Bragg energy is near $12.4 \mathrm{keV}$. Part (a) plots the reflection (solid, red) and transmission (blue dotted) curves for a "thick" crystal with $d=$ $150 \mu \mathrm{m}$, demonstrating no transmission with a slightly asymmetric reflection curve within the crystal bandpass. Outside the bandpass, the reflection curve oscillates about the absorptionfree, $d \rightarrow \infty$ curve (green, dashed line). Graph (b) plots the same curves for a "thin" crystal with $d=42 \mu \mathrm{m}$. The thin crystal reflectivity curve is rounded and decreased from the semi-infinite case, while the transmission $|t|^{2} \approx 4.5 \%$ at $\delta E=0$. The complex phase of (a) and (b) is nearly identical to the ideal case (8) near the origin $\delta E=0$.

Beyond these differences leading to absorption and transmission, the general reflection characteristics of physically realizable Bragg crystals are very similar to those discussed in the previous section: namely, suitably oriented crystals exhibit a very high reflectivity in a narrow frequency range (the Darwin width), and, within this bandpass, the crystal imparts a complex phase shift to the radiation whose lowest-order effect is to delay the reflected pulse in time. In terms of FEL performance, the photoabsorptive losses place limits on the minimal FEL gain required for net amplification, while the complex phase shift and its corresponding delay requires a precise, micron-scale shift in the resonant cavity length.

Crystals that are sufficiently thick (typically $d \gtrsim$ $100 \mu \mathrm{m}$ for $E \lesssim 15 \mathrm{keV}$ ) exhibit vanishingly small transmission within the Darwin width. The absorption in the Darwin width is typically a few percent for diamond, and is a slightly asymmetric function of photon energy, which creates a slightly asymmetric reflectivity curve. Nevertheless, the qualitative features of the complex reflectivity remain quite similar to those presented in the previous section. As an illustration, we plot the response of a thick $(d=150 \mu \mathrm{m})$ diamond $[\mathrm{C}(4,4,4)]$ crystal in Fig. 3(a), which illustrates the decrease in peak reflectivity, as well as an asymmetric response in $|r|^{2}$ and $|t|^{2}$ due to (in this case) higher absorption for $\delta E<0$. For photon energies outside the Darwin width, the phase factors in (3) give rise to oscillations in the complex reflectivity $r$ and transmittance $t$, evidence of which can also be seen in Fig. 3(a). 
Finally, we note that the complex phase of $r$ is nearly indistinguishable from its ideal (i.e., semi-infinite, absorption-free) counterpart near $\delta E=0$. In this way, an incident pulse whose bandwidth is well contained within the Darwin width is reflected with a slight loss of intensity, a delay in time, and with minimal distortion. Thus, we can again use an appropriate optical cavity length decrease to compensate for most of the crystal-induced dispersion.

Thin crystals can be employed for partial transmission of the cavity radiation. We plot $|r|^{2}$ and $|t|^{2}$ in Fig. 3(b), where we use the diamond parameters of Fig. 3(a), but with a decreased thickness $d=42 \mu \mathrm{m}$ that is chosen to couple $\sim 4.5 \%$ of the radiation out of the cavity. We see that the thin crystal response is smoothed within the Darwin width, while the oscillations outside this region have a decreased frequency in $\delta E$. Nevertheless, near $\delta E=0$, the absorption for the thick and thin crystals are nearly identical, as is the complex phase of $r$. Thus, the thin crystal behaves much like the thick crystal described above but with a lower reflectivity due to the nonzero transmission.

\section{FEL THEORY AND SIMULATION TECHNIQUES}

In this section, we review a few basic theoretical results and describe the additions to the GINGER code that have been done to simulate the x-ray FEL oscillator. We note how the Bragg crystals affect the single-pass gain of the FEL in III A, while explaining the basic transverse profile of the radiation in III C. These two pieces of physics will be useful to interpret the simulation results to be presented in Sec. IV. While the 2D code GINGER used for these simulations is fairly well known and solves the FEL equations within a numerical framework that has a long history, the inclusion of the Bragg crystal elements is somewhat new, and we summarize our method for implementing this portion of the oscillator in Sec. III D.

\section{A. Single-pass gain in the x-ray FEL oscillator}

In order for the radiation in the FEL oscillator to grow, we require that the net gain in any pass exceeds the losses, i.e., that

$$
R(1+G)>1,
$$

where $R$ is the total reflectivity of the crystals and mirrors, and $G$ is the net gain per pass. In general, $G$ can be reduced from its ideal FEL value $G_{0}$ by many effects including slippage, finite electron beam length, cavity length/timing errors, etc. For the x-ray FEL oscillator, however, the dominant gain reduction is typically due to the small spectral acceptance of the Bragg crystal. For a crystal with rms spectral bandpass given by $\sigma_{\omega}$, the reduction in net gain from $G_{0} \rightarrow G$ due to the finite crystal acceptance can be analytically calculated in both the low-gain [4] and high-gain [8] limit:

$$
G= \begin{cases}G_{0}-\frac{\sqrt{G_{0}}}{2 \sigma_{t} \sigma_{\omega}} & \text { if } G_{0} \ll 1 \\ G_{0} \frac{\sqrt{1+8 \sigma_{t}^{2} \sigma_{\omega}^{2}}-1}{\sqrt{1+8 \sigma_{t}^{2} \sigma_{\omega}^{2}}+1} & \text { if } G_{0} \gg 1,\end{cases}
$$

where $\sigma_{t}$ is the rms length of the electron beam. Both of these formulas have $G \rightarrow G_{0}$ in the limit of $\sigma_{t} \sigma_{\omega} \rightarrow \infty$, and show that the gain decreases from its nominal value $G_{0}$ as the electron beam becomes shorter. Physically, this arises because only the fraction of the current whose spectral content lies within the crystal bandpass effectively contributes to the gain. This gain reduction is significant when the electron beam length becomes less than the inverse bandwidth of the crystal, i.e., when $\sigma_{t} \sigma_{\omega} \lesssim 1$. For a diamond crystal at $12 \mathrm{keV}$, the rms bandwidth $\sigma_{\omega} \approx$ $12 \times 10^{12} \mathrm{rad} / \mathrm{s}=12 \mathrm{ps}^{-1}$. Thus, we expect this gain reduction effect should become an important consideration for sub-ps electron beams.

As discussed in Sec. II A, the complex reflectivity also induces a phase shift on the radiation that is approximately linear in the energy (or frequency) in the Darwin width. We can model this affect in the low-gain regime by using supermode theory $[4,9,10]$, approximating the phase shift by $\phi=\omega \ell / c$, where $\ell$ is proportional to the crystal penetration length. As shown in Sec. II A, each crystal contributes approximately twice its penetration length to $\ell$. Including both this crystal dispersion and a cavity length change $\Delta z$, we find that

$$
G=G_{0}-2 \sigma_{\omega}^{2}(\ell+\Delta z)^{2} / c^{2}-\frac{\sqrt{G_{0}}}{2 \sigma_{t} \sigma_{\omega}} .
$$

The final term is the gain reduction due to the finite crystal bandpass, while the second term shows that we should reduce the cavity length by setting $\Delta z=-\ell$ to within a few percent of the inverse bandpass $1 / \sigma_{\omega}$ so as to not adversely reduce the gain.

\section{B. X-ray cavity configurations}

To trap the radiation and maximize the FEL gain, the $\mathrm{x}$-ray cavity must provide appropriate transverse focusing of the radiation. This can be done with presently available technology by using Bragg crystals to reflect the radiation and grazing incidence mirrors to provide the $\mathrm{x}$-ray focusing, as shown in Fig. 4(a). Crystal A would be chosen to be sufficiently thin so as to couple $\sim 5 \%$ of the radiation out of the cavity, while the grazing incidence mirrors $\mathrm{M}_{1}$ and $\mathrm{M}_{2}$ would provide the x-ray focusing and angular collimation.

To have a reflectivity near unity, the grazing incidence angle must be less than the critical angle $\sim 1 \mathrm{mrad}$, limiting $\Theta \lesssim 1 \mathrm{mrad}$ in the two-crystal cavity. From Bragg's law $\lambda=\lambda_{H} \cos \Theta$, we see that the limits imposed on $\Theta$ by the two-crystal cavity severely restrict the range of radiation wavelength. To circumvent this problem, Kim and Shvyd'ko [11] proposed the four-crystal FEL oscillator cavity of Fig. 4(b), for which the incidence angle can be 

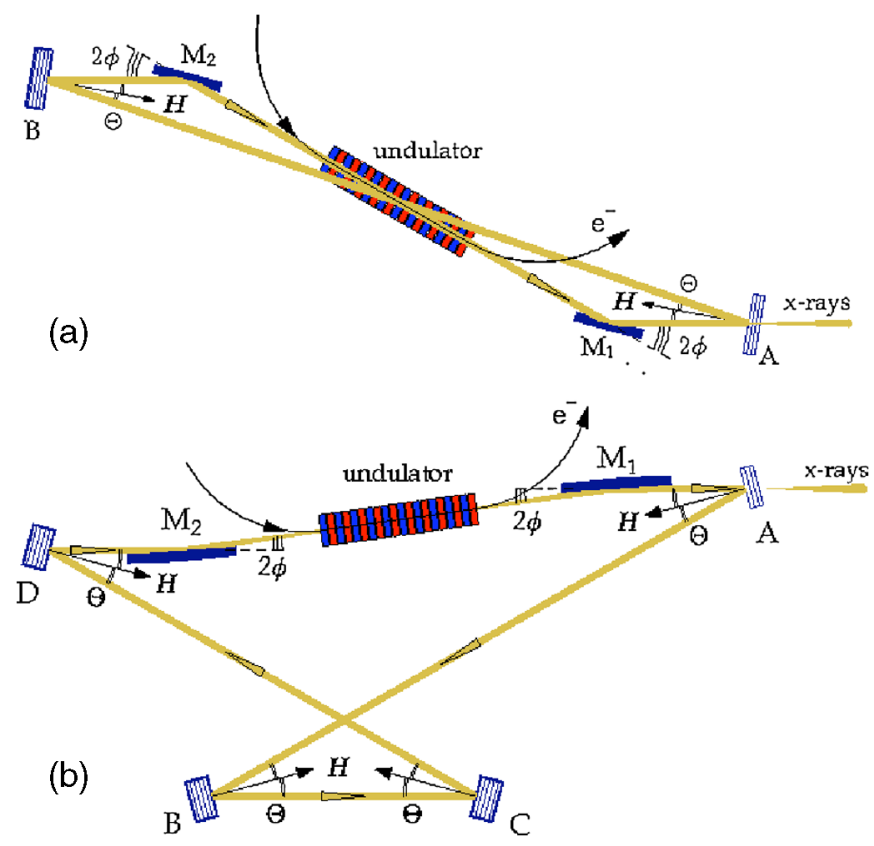

FIG. 4. X-ray cavity schemes. Part (a) uses two crystals to trap the radiation and two grazing incidence mirrors to provide focusing and angular collimation. The x-ray FEL oscillator can be made tunable by using four crystals as shown in (b). The angle of incidence $\Theta$ can be varied while simultaneously adjusting the cavity length to change the photon energy while keeping the round-trip time fixed.

adjusted to tune the photon energy over a fairly wide range. We will present results of the performance of both cavity configurations in Sec. IV.

\section{Transverse resonator physics}

Since the gain $G_{0} \lesssim 2$, the transverse mode is typically well described by the gain-free, vacuum resonator mode of the cavity. In order to understand the transverse $\mathrm{x}$-ray profile, we consider the symmetric two-mirror resonator cavity shown in Fig. 5. This cavity is a simple model of the two-crystal configuration in Fig. 4(a). The two-mirror resonator cavity has stable, trapped vacuum modes when (see, e.g., [12])

$$
f=\frac{L_{c}}{4}+\frac{Z_{R}^{2}}{L_{c}}
$$

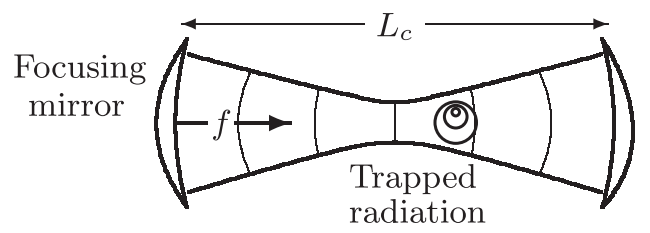

FIG. 5. Schematic of a simple two-mirror resonator cavity. The transverse properties of the stable vacuum mode are nearly identical to that of the growing FEL radiation simulated in this paper. where $f$ is the mirror focal length, $L_{c}$ is the length of the cavity, and $Z_{R}=4 \pi \sigma_{x}^{2} / \lambda$ is the Rayleigh range for a radiation beam with rms transverse size $\sigma_{x}$. Although the relationship (16) is only strictly valid in the low-gain limit, $G_{0} \ll 1$, we have found that the transverse waist and Rayleigh range of the growing mode closely follow Eq. (16) for all the cases presented in this paper, some of which have nominal FEL gains $G_{0} \sim 2$.

To set the parameters of (16), we require that the cavity length be chosen so that the round-trip time of the photons matches the spacing between electron bunches, which for a repetition rate between $1-2 \mathrm{MHz}$ implies that $L_{c} \sim$ $75-150 \mathrm{~m}$. We choose the $\mathrm{x}$-ray Rayleigh range so as to maximize the FEL gain, which occurs when $Z_{R}$ matches the vacuum focusing of the electron beam and is of order $L_{u} / 2 \pi$, i.e., when $Z_{R} \approx \sigma_{x}^{2} / \varepsilon_{x} \approx L_{u} / 2 \pi$, with $\sigma_{x}$ the electron beam waist, $\varepsilon_{x}$ its emittance, and $L_{u}$ the length of the undulator [13]. Using expression (16) yields the mirror focal length.

To study more general cavity configurations like the four-crystal cavity of Fig. 4(b), we use the paraxial approximation to study the transverse $\mathrm{x}$-ray cavity modes. A convenient way to do this is to introduce a distribution of fictitious rays in the position $(x)$ and angle $\left(x^{\prime}\right)$ phase space [14]. Since the rays transform in the same way as in geometric optics, we can make use of the familiar matrix formalism in particle optics $[15,16]$. Although the distribution is not a genuine probability distribution of physical rays (it is a Wigner distribution; see, e.g., [17]), physically measurable quantities, such as the density or angular profile, can be obtained by integrating over the variables not measured. In this approach, a transversely coherent mode has a Gaussian profile with rms emittance given by the radiation wavelength $\lambda$ via $\varepsilon_{r}=\lambda / 4 \pi$. At the mode waist, the $x$ - $x^{\prime}$ correlation vanishes, $\left\langle x x^{\prime}\right\rangle=0$, where the angular brackets indicate averaging over the ray distribution. The Rayleigh length $Z_{R}$ at a waist is related to the rms size and angular divergence as follows:

$$
\left\langle x^{2}\right\rangle=\varepsilon_{r} Z_{R}, \quad\left\langle x^{\prime 2}\right\rangle=\varepsilon_{r} / Z_{R},
$$

and the Rayleigh length is equivalent to the CourantSnyder beta function in the terminology of particle optics [15]. Although we have made reference to rays in our description, it can be shown that the matrix formulation is equivalent to Fresnel wave propagation for Gaussian waves (see, e.g., Siegman [12]). In the laser community this approach is usually referred to as the $A B C D$-matrix method, and the matrix transformations we list can be used to derive the stable Rayleigh range and wave front curvature for Gaussian waves.

To study the four-crystal cavity of Fig. 4(b), we note that this configuration is equivalent to the linear periodic system shown in Fig. 6 assuming that the role of crystals is solely to deflect the ray. In Fig. 6 , the focusing mirrors $\mathrm{M}_{1}$ and $\mathrm{M}_{2}$ have focal length $f$, we denote the consecutive 


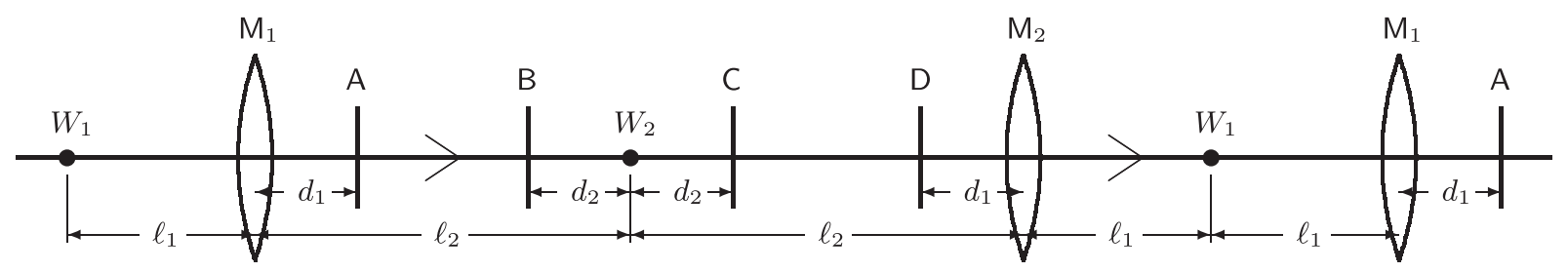

FIG. 6. A schematic of the optical cavity equivalent to that of Fig. 4(b), but unfolded to straighten the optical axis. A, B, C, and D label the crystal positions, while $W_{1}\left(W_{2}\right)$ indicate the position of the radiation waist inside (outside) the undulator.

crystals A, B, C, and D as we did in Fig. 4(b), and we have additionally labeled the relevant cavity distances. Note that $W_{1}$ is the position of the waist at the middle of the undulator, while the other waist is located at $W_{2}$. Introducing the notation

$$
\mathrm{L}(\ell)=\left[\begin{array}{ll}
1 & \ell \\
0 & 1
\end{array}\right] \quad \mathrm{F}=\left[\begin{array}{cc}
1 & 0 \\
-1 / f & 1
\end{array}\right] \quad X_{i} \equiv 1-\frac{\ell_{i}}{f},
$$

the criteria for stability can be determined by evaluating the matrix for one full period. The system is stable provided that $0<X_{1} X_{2}<1$; consequently, $X_{1}$ and $X_{2}$ should have the same sign.

The tolerance in the orientation of the crystals can be determined using a standard method from accelerator physics. Suppose the crystal $\mathrm{A}$ is oriented at an angle $\Delta \theta$ from the ideal angle, so that an on-axis ray is deflected by an angle $2 \Delta \theta$. The coordinates of the displaced optical axis at $W_{1}$ can be found by requiring that the displaced reference trajectory is periodic with the period of the lens system:

$$
\begin{aligned}
{\left[\begin{array}{c}
\Delta x \\
\Delta x^{\prime}
\end{array}\right]=} & \mathrm{L}\left(\ell_{1}\right) \operatorname{FL}\left(2 \ell_{2}\right) \operatorname{FL}\left(\ell_{1}\right)\left[\begin{array}{c}
\Delta x \\
\Delta x^{\prime}
\end{array}\right] \\
& +\mathrm{L}\left(\ell_{1}\right) \mathrm{FL}\left(2 \ell_{2}-d_{1}\right)\left[\begin{array}{c}
0 \\
2 \Delta \theta
\end{array}\right]
\end{aligned}
$$

The first line has the full round-trip transformation from the waist $W_{1}$ at the undulator center, while the second line includes the angular error due to crystal $A$.

Let us now consider the physical parameters of the x-ray FEL oscillator described in Ref. [4] but employing the four-crystal cavity of Fig. 6. We choose the Rayleigh range inside the undulator $Z_{1}=10 \mathrm{~m}$ to maximize the gain of the FEL centered at $W_{1}$. Choosing a large value of the Rayleigh range $Z_{2}$ after the focusing elements is desirable so that the angular divergence seen by the crystals is small. We choose $Z_{2}=250 \mathrm{~m}$ so that the angular divergence around $W_{2}$ including the two lenses is 5 times smaller than the value at $W_{1}$. We also choose $X_{1} X_{2}=0.01$ so that the cavity is sufficiently far from the region of instability, while the cavity geometry sets the final relationship:

$$
d_{1}+d_{2}=\frac{\ell_{2}^{2}-\ell_{1}^{2}-H^{2}}{2\left(\ell_{1}+\ell_{2}\right)},
$$

where $H$ is the (typically small) vertical distance between the undulator and the line joining crystals $\mathrm{B}$ and $\mathrm{C}$ and in Fig. 4(b). If we assume that $H=d_{2}=1 \mathrm{~m}$, then the coordinates of the displaced waist are

$$
\left[\begin{array}{c}
\Delta x \\
\Delta x^{\prime}
\end{array}\right]=\left[\begin{array}{c}
51.257 \\
1.273
\end{array}\right] \Delta \theta
$$

We require the displacements in position and angle to be less than one-tenth of the mode size and angular divergence, which are $10 \mu \mathrm{m}$ and $1 \mu \mathrm{rad}$, respectively. Using the positional requirement $\Delta x<1 \mu \mathrm{m}$, we find that $\Delta \theta<20 \mathrm{nrad}$; the tolerance obtained using the angular requirement (i.e., $\Delta x^{\prime}<0.1 \mu \mathrm{rad}$ ) is much less stringent. To improve and extend this theory, we are in the process of performing a more detailed tolerance study using more general cavity configurations including multiple misalignments, variation of the radiation wavelength, and the effects of asymmetric Bragg crystals [18,19].

\section{GINGER implementation of the Bragg crystal reflectivity}

As mentioned earlier, GINGER is an axisymmetric $(r-z)$, polychromatic FEL simulation code that has been used previously on many single-pass amplifier and multipass oscillator problems of interest. The oscillator physics package was originally formulated in the mid-1990s (see, e.g., [20]) and supplements the standard single-pass FEL amplifier formulation with a Huygens integral method (see Eq. 16.94 in [12]) to transport the outgoing FEL radiation from the undulator exit to the various mirror surfaces and back to the undulator entrance. This linear evolution outside the undulator is represented numerically in GINGER with the complex propagation matrix $\mathcal{T}$. The matrix elements $\mathcal{T}_{i j}$ are calculated once at the beginning of the simulation, after which the electric field at the start of any pass is related to that at the end of the previous pass by the relationship

$$
E_{\text {new }}\left(r_{i}\right)=\sum_{j} \mathcal{T}_{i j} E_{\text {exit }}\left(r_{j}\right),
$$

where index $i$ refers to undulator entrance and $j$ to undulator exit. The matrix $\mathcal{T}$ includes effects due to mirror curvature, mirror holes, and free space propagation. Imperfect reflectivity (i.e., $R<1$ ) is currently presumed to have a constant value over the mirror surfaces. GINGER 
uses a radially nonuniform grid for the FEL radiation calculations; the intermediate gridding on the mirror surfaces is also typically nonuniform in order to maximize computational efficiency and accuracy. GINGER also uses the eikonal approximation (also known as either the slowly varying envelope approximation or the paraxial approximation) and presumes that diffraction and, before the changes listed below, the cavity reflectivity are independent of wavelength over the narrow spectral bandpass modeled.

For the x-ray FEL oscillator simulations presented here, a number of changes and improvements were needed in GINGER. First, because typical Fresnel numbers are so large when considering propagation of Angstrom-wavelength radiation, we found that the original Huygens-Fesnel integral algorithm was on occasion numerically too inaccurate, especially for very low-gain systems. We corrected this problem by using the identity $J_{0}(x) \equiv\left[H_{0}^{(1)}(x)+\right.$ $\left.H_{0}^{(2)}(x)\right] / 2$ in the Fresnel integration kernel for $x \geq 3$, where $H_{0}$ is the Hankel function. For large $x$, this change isolates the fast oscillation term of $J_{0}$, simplifying the numerical integration while improving its accuracy for a given radial gridding. This approach was suggested previously in [21].

The second change was made necessary by the $\lambda$-dependent complex reflectivity of the Bragg crystals because normally the GINGER code operates completely in the time domain, with all spectral decomposition done only after completion of the simulation by a separate postprocessor code. Implementation of the $\lambda$-dependent crystal reflectivity requires a Fourier transform of $E(t)$ to be done at each radial grid zone after each oscillator pass, followed by a multiplication of $E(\lambda)$ by the complex reflectivity $R(\lambda)$ for each crystal surface, after which the result is transformed back to the time domain. Since this is done only once per pass, the computational cost is fairly negligible. The complex $R(\lambda)$ is read in as a simple lookup table at the beginning of the simulation with linear interpolation done between table values. For the simulation results presented here, we also presumed that the radiation divergence is much smaller than the angular acceptance of the Bragg crystal; this permits the use of a single reflectivity function $R(\lambda)$.

\section{X-RAY FEL OSCILLATOR SIMULATIONS}

In this section we discuss possible electron beam, undulator, and Bragg crystal properties suitable for x-ray production at several different photon energies, and present simulation results from the 2D code GINGER indicating that such devices can produce $\sim 10^{9}$ coherent photons in a $0.1-$ 1.0 ps pulse whose peak power is of order a few MW. Typical beam and undulator parameters are listed in the top portion of Table I, where we have fixed the transverse emittance $\varepsilon_{x}=0.2 \mathrm{~mm} \mathrm{mrad}$, the energy spread $\sigma_{\mathrm{E}}=$ $1.4 \mathrm{MeV}$, and the electron bunch length $\sigma_{t}=1 \mathrm{ps}$. The remaining parameters were adjusted to ensure that the single-pass net gain $G$ moderately overcame the losses, i.e., $(1+G) R>1$. Note that for these cases we have $1 / \sigma_{t} \sigma_{\omega} \ll 1$ so the decrease in the ideal single-pass FEL gain $G_{0}$ from (14) is small; when the beam length approaches that associated with the inverse bandwidth of the Bragg mirror the decrease in $G$ must be compensated

TABLE I. Possible undulator, beam, and optical cavity parameters. For all cases the transverse emittance $\varepsilon_{x}=0.2 \mathrm{~mm}$ mrad, energy spread $\sigma_{\mathrm{E}}=1.4 \mathrm{MeV}$, the rms beam length $\sigma_{t}=1 \mathrm{ps}$, while the undulator gap is $5 \mathrm{~mm}$. The characteristics in the lower box are from x-ray pulses that have been coupled out of the cavity through the thin crystal; for a Gaussian $\sigma_{\tau} \sigma_{\omega}(\mathrm{rms})=1 / 2$.

\begin{tabular}{lccccc}
\hline \hline Parameter & $4.9156 \mathrm{keV}$ & $5.591 \mathrm{keV}$ & $12.04 \mathrm{keV}$ & $14.326 \mathrm{keV}$ & $19.936 \mathrm{keV}$ \\
\hline$\lambda_{u}(\mathrm{~cm})$ & 2.244 & 1.96 & 1.76 & 1.656 & 1.50 \\
$N_{u}$ & 1000 & 1500 & 3000 & 3000 & 3000 \\
FEL $K$ & 2.50 & 1.53 & 1.51 & 1.322 & 1.05 \\
$E_{\text {beam }}(\mathrm{GeV})$ & 7.0 & 5.0 & 7.0 & 7.0 & 7.0 \\
$I_{\text {peak }}(\mathrm{A})$ & 10.0 & 20.0 & 10.0 & 20.0 & 20.0 \\
$Z_{\beta}(\mathrm{m})$ & 4.5 & 6.0 & 10.0 & 10.0 & 10.0 \\
$G_{\text {linear }}$ & 0.32 & 0.60 & 0.36 & 0.55 & 0.32 \\
$R_{\text {total }}$ & 0.84 & 0.66 & 0.85 & 0.80 & 0.85 \\
$L_{\text {cavity }}(\mathrm{m})$ & 40.0 & 60.0 & 100.0 & 100.0 & 100.0 \\
Bragg crystal & $\mathrm{C}(220)$ & $\mathrm{Si}(224)$ & $\mathrm{C}(444)$ & $\mathrm{Al}_{2} \mathrm{O}_{3}(00030)$ & $\mathrm{C}(559)$ \\
Crystal absorption & $5 \%$ & $12 \%$ & $1.4 \%$ & $12 \%$ & $0.9 \%$ \\
$P_{\text {sat }}(\mathrm{MW})$ & 99.0 & 22.7 & 25.8 & 25.2 & 12.9 \\
\hline Spectral FWHM (meV) & 2.67 & 2.70 & 1.95 & 2.25 & 1.95 \\
Temporal FWHM (ps) & 1.68 & 1.35 & 1.58 & 1.94 & 1.30 \\
$\sigma_{\tau} \sigma_{\omega}(\mathrm{rms})$ & 2.25 & 1.38 & 0.98 & 3.98 & 1.14 \\
Photons/pulse & $4.6 \times 10^{9}$ & $6.0 \times 10^{8}$ & $1.1 \times 10^{9}$ & $6.2 \times 10^{8}$ & $3.6 \times 10^{8}$ \\
Peak power (MW) & 2.5 & 1.5 & 1.66 & 0.76 & 0.57 \\
\hline \hline
\end{tabular}


for by increasing $G_{0}$. We discuss such a scenario in Sec. IV B, in which we show that, by compressing the low-charge beam to $100 \mathrm{~A}$ and $100 \mathrm{fs}$, the FEL gain can be increased so that $G>1$, which in turn leads to less stringent conditions on the reflectivity $R$ and, potentially, on the cavity alignment.

The properties of diamond, including high peak reflectivity, low absorption, high thermal conductivity, and small coefficients of thermal expansion, make it an ideal material for high-power x-ray Bragg mirrors. Although large-scale, perfect diamond crystals have yet to be produced, the FEL oscillator only requires a small $\sim 100 \mu \mathrm{m}^{2}$ region to be defect free, which seems to be a realistic possibility [22]. Nevertheless, we also consider alternatives in Table I, including silicon and sapphire mirrors. While perfect silicon crystals are readily available, they typically have large photoabsorption that decreases the net reflectivity while also increasing the heat load on the crystal. This may lead to further degradation in cavity performance due to large temperature gradients or damage in the crystal. Regions of perfect sapphire crystals have been produced, although, at the $\mathrm{x}$-ray powers we envision, they too may suffer from damaging heat loads. We list some possible x-ray FEL oscillator parameters in Table I, including undulator, beam, and optical cavity parameters for five different radiation wavelengths.

The values in Table I reflect the wide range of parameters that can potentially generate intense $\mathrm{x}$ rays in a FEL oscillator but are by no means exhaustive. One significant difficulty that has yet to be addressed involves the heat load and damage threshold of the Bragg mirrors. While we expect material damage to be negligible at these powers and wavelengths, the heat load will increase the mirror temperature, which may subsequently shift the resonant wavelength for Bragg diffraction $\lambda \approx 2 d \cos \Theta$ through thermal expansion of the crystalline plane spacing $d$. Because of their relatively high levels of absorption, this may limit the utility of silicon or sapphire Bragg mirrors. On the other hand, the small levels of absorption $(\sim 1 \%)$, small coefficients of thermal expansion $\left(\sim 3 \times 10^{-8} 1 / \mathrm{K}\right.$ at $100 \mathrm{~K}$ [23]), and high heat conductivities make perfect diamond crystals an ideal candidate for the x-ray FEL oscillator, and our subsequent examples will focus on it.

\section{A. 12-keV FEL using diamond Bragg mirrors}

An x-ray FEL operating near $1 \AA$ with the parameters listed in the third column of Table I has a linear gain $G \approx$ $36 \%$, while the theoretical peak intensity reflectivity of the two mirrors is $94 \%$. Note that the gain reduction due to the finite temporal e-beam length and the narrow crystal bandwidth for a 1 ps pulse incident on the $\mathrm{C}(444)$ reflection is small, $\sqrt{G_{0}} / \sigma_{t} \sigma_{\omega} \approx 0.02$. Additionally, we assume that there are two focusing elements whose effective focal length is $52.0 \mathrm{~m}$, and that each element induces an additional loss of $5 \%$, so that the net per-pass gain is

$$
(1+G) R \approx 1.14>1 .
$$

We show a typical example of the radiation evolution from shot noise initiation through saturation to the development of a steady state in Fig. 7. The first row of panels displays the temporal power profile after various pass numbers using a solid, red line; we include the electron beam current profile as the green, dashed line for reference. The second row shows the cavity pulse spectrum after the same pass number with the solid red line, with the total crystal and mirror reflectivity bandpass included as the dashed green line. These plots indicate significant temporal and spectral fluctuations during the initial seeding and exponential growth phase for $N_{\text {pass }} \lesssim 150$. After saturation the $\mathrm{x}$-ray power is nearly single peaked in both time and energy, with the temporal width fixed by the electron beam to be of order 1 ps. Nevertheless, slow evolution proceeds over the next several hundred passes, until the temporal and spectral profiles reach a steady state. The final panel plots the power and spectra at $N_{\text {pass }}=600$ and $N_{\text {pass }}=$ 1000 , showing very close overlap.

The temporal and spectral power profiles of the outcoupled x-ray pulse after the 1000th pass are shown in Fig. 8. To obtain these plots, we applied the complex transmission coefficient of the thin crystal to the complex electric field. Comparison to the final panels of Fig. 7 demonstrate that the output pulse properties are nearly identical to that of the cavity pulse, the only significant distinguishing feature being the $\sim 95 \%$ decrease in total energy due to the small transmission of the thin crystal.

After 1000 passes, the transmitted radiation spectrum has a measured rms width of $1.29 \mathrm{meV}$, while the temporal rms width is about $0.51 \mathrm{ps}$. This corresponds to a bandwidthtemporal product of 0.98 , approaching the Fourier limit of 0.5 for a Gaussian pulse profile. The output pulse has a peak power of $1.54 \mathrm{MW}$, and the total energy coupled out of the cavity is $2.2 \mu \mathrm{J}$, corresponding to $1.1 \times 10^{9}$ photons.

\section{B. Relaxed operation using a $\mathbf{1 0 0}$ fs electron beam}

The "canonical" examples discussed thus far use a 1 ps electron beam with 25 to $50 \mathrm{pC}$ of total charge and a peak current between 10-20 A. This case typically requires $\sim 3000$ undulator periods, and a beam with normalized emittance of $\varepsilon_{x}=0.2 \mathrm{~mm} \mathrm{mrad}$ and normalized energy spread $\sigma_{E} / E=0.02 \%$ to overcome the assumed $85 \%$ total cavity loss and yield net FEL gain. In this section, we consider the possibility of further compressing a $25 \mathrm{pC}$ electron beam to $100 \mathrm{fs}$, raising the peak current to $100 \mathrm{~A}$. We will find that such a compressed beam increases the single-pass FEL gain, thereby relaxing the requirements on undulator length, beam emittance, and/or total round-trip reflectivity. We will discuss a few such specific examples at $12-\mathrm{keV}$ photon energy, although similar results will also hold for the other 5-, 14-, and 20-keV examples discussed in the previous sections. 

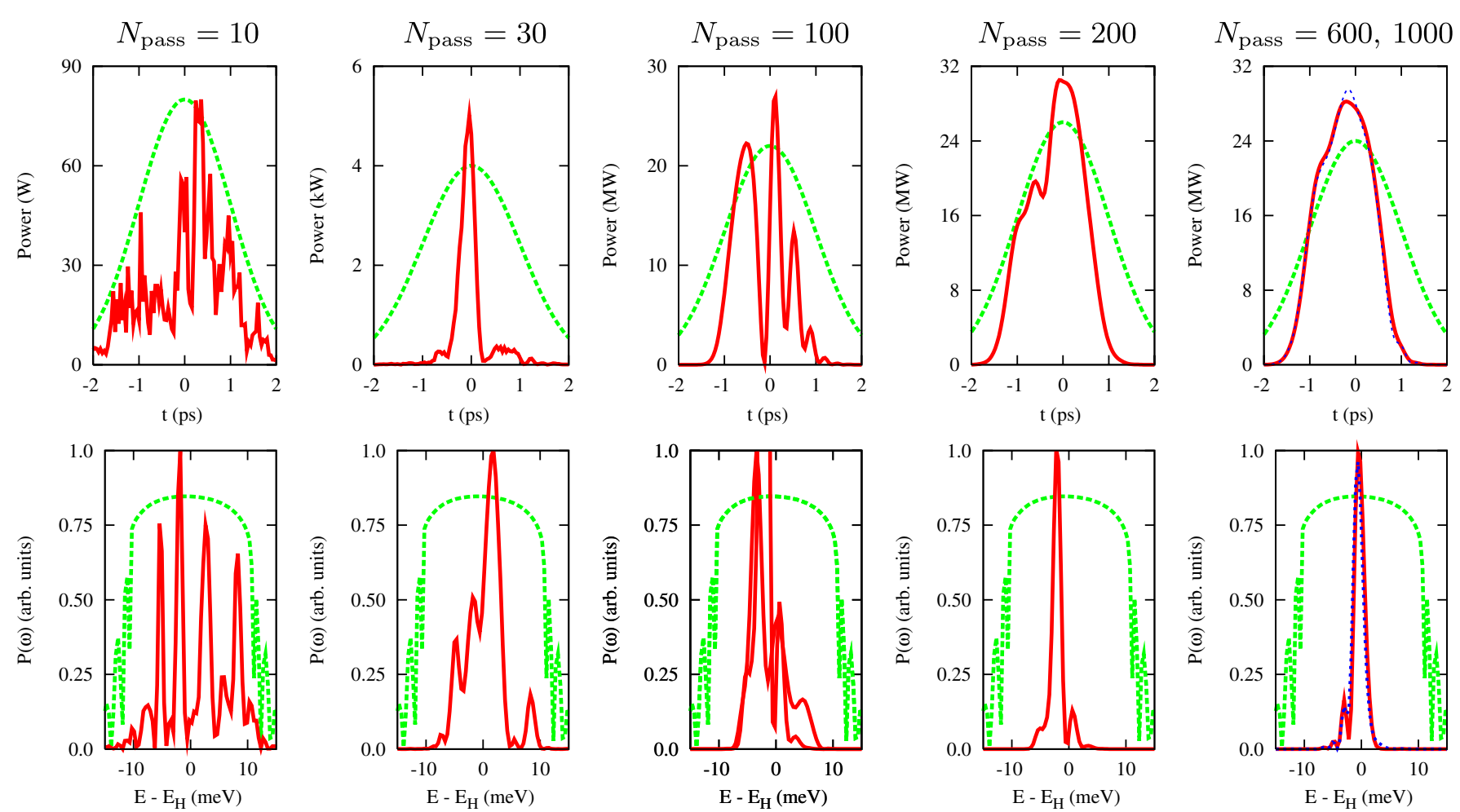

FIG. 7. Snapshots of the trapped cavity radiation for a typical simulation of the $\mathrm{x}$-ray FEL oscillator at $1 \AA$ (parameters are listed in Table I). The top row shows the transverse-averaged power in solid (red) lines, with the 1 ps electron beam current profile displayed by the dotted (green) line for comparison. The bottom row plots the normalized radiation spectrum with the solid (red) line, and includes the total reflectivity of the Bragg crystals (including the artificial decrease to 85\% total reflectivity) as the dotted (green) line. The time and spectral properties fluctuate significantly until saturation around 200 passes, after which a slow evolution occurs until a nearequilibrium state is reached around pass number 500. The final panel includes both power and spectral profiles at both 600 and 1000 passes, which are nearly identical. After pass 1000, the temporal and spectral properties of the transmitted radiation are nearly identical to that in the cavity, with the only difference being a decrease in the energy/power of the output to $\sim 4 \%-5 \%$ of the cavity pulse.

For a $100 \mathrm{~A}, 100 \mathrm{fs}$ electron beam, we list the net FEL gain $G$, which includes the decrease due to the finite spectral bandwidth of the Bragg crystals, for several different beam and undulator parameters in Table II. For the present discussion we assume that the total round-trip loss equals $50 \%$; note that the number of undulator periods has been chosen such that $1+G>2$, so that the net gain
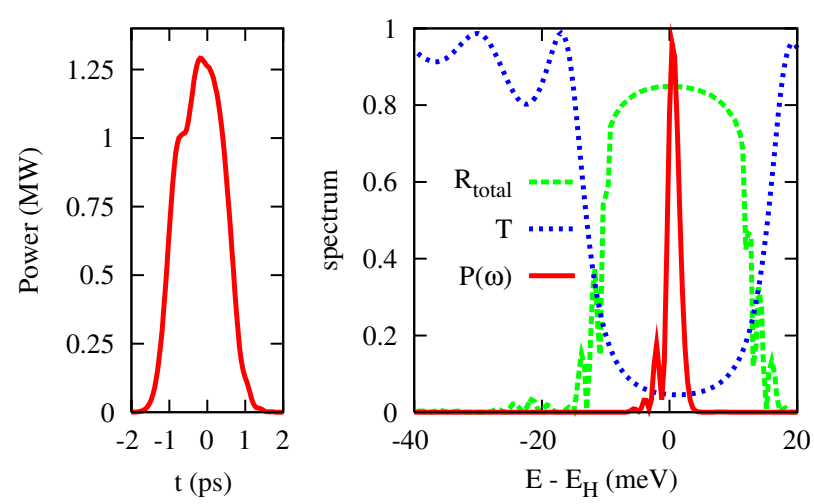

FIG. 8. Outcoupled pulse power profiles in time and energy after 1000 oscillator passes; compare with the cavity pulses plotted in the last panel of Fig. 7. exceeds the total loss. Table II indicates that raising the current by a factor of 10 at fixed charge can result in relaxed requirements for the beam quality and/or the undulator length, even with the comparatively low quality factor of the cavity. The output pulse peak power is similar or greater than similar parameters at 10 A peak current, although the photon number is reduced due to the shorter $\mathrm{x}$-ray pulse, whose width is $\sim 150 \mathrm{fs}$ full-width at halfmaximum (FWHM). Since the temporal width has decreased by a factor of 15-20, the spectrum has broadened a similar amount, to a FWHM $\sim 15-18 \mathrm{meV}$.

To compare with the 1 ps case of Figs. 7 and 8, we show the output pulse power profile in time and energy in Fig. 9, where we take the beam to have $\varepsilon_{x}=0.3 \mathrm{~mm} \mathrm{mrad}$ and $\sigma_{\mathrm{E}} / E=0.02 \%$ from Table II. The main peak is nearly Gaussian in shape but now is followed in time by a series of trailing pulses of decreasing amplitude. These pulses can be associated with the multiple reflected pulses at the crystal surfaces; their $\sim 300$ fs periodicity can be roughly associated to twice the thickness of the thin crystal, $2 d=$ $84 \mu \mathrm{m}$. Because of these trailing pulses, the rms width is somewhat larger than what one might expect for a Gaussian. However, since they come after the main pulse, 

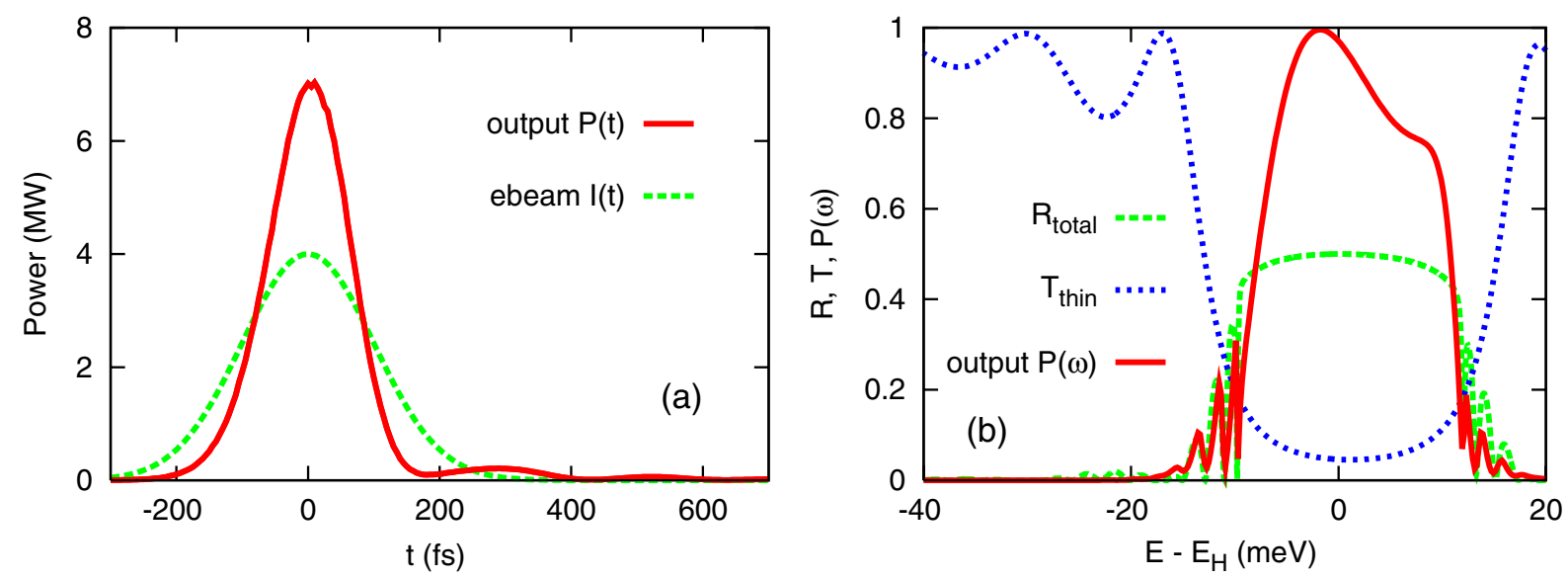

FIG. 9. Time and spectral profiles of the output radiation using a $100 \mathrm{fs}$ electron beam and the parameters listed in Table II. In panel (a), the temporal profile shows $\sim 7 \mathrm{MW}$ peak power in a nearly Gaussian first pulse; the subsequent pulse train is related to multiple reflections from the crystal surface. Panel (b) shows the spectral properties of the cavity reflectivity $R_{\text {total }}$ with the dashed (green) line and the transmission with the dotted (blue) line. The spectrum of the cavity pulse just fits within the crystal bandwidth, which leads to a similar shaped transmitted spectrum shown by the solid (red) line. Because the transmission of the thin crystal increases near the edges, the transmitted pulse spectrum is slightly distorted; nevertheless the spectrum is nearly single peaked, with a FWHM $\sim 18$ meV and a fractional bandwidth $\sim 1.5 \times 10^{-6}$.

the prepulse is minimal, and the $\mathrm{x}$-ray burst is expected to have a high contrast ratio $\sim 10^{6}$ at the leading edge. Figure 9 also plots the corresponding outcoupled spectral profile. Because the bandwidth of the short $100 \mathrm{fs}$ electron beam is greater than that of the crystals, the $\mathrm{x}$-ray spectrum fills the Bragg bandpass with a single peak. The slight distortion in the spectrum near $10 \mathrm{meV}$ arises from the increased transmission of the thin crystal (plotted as the dotted blue line) away from the central energy.

\section{Four-crystal geometry for a tunable, low-bandwidth $x$-ray source}

The $x$-ray FEL oscillator scenarios presented in the two previous sections used the simple two-crystal cavity

TABLE II. Electron beam, undulator, and output radiation parameters for the $100 \mathrm{fs}, 100 \mathrm{~A}$ operation at $1 \AA$. For net gain and our definition of the gain $G$ and total reflectivity $R$, we require $R(1+G)>1$; the parameters listed assume $R=0.5$. The multiple reflections from the crystal surfaces lead to a sequence of low-amplitude pulses, as shown in Fig. 9, although the first, main pulse is nearly Gaussian. Note that these oscillations follow the main pulse, so that the leading, main pulse has a high contrast ratio with minimal prepulse pedestal.

\begin{tabular}{lcccc}
\hline \hline$\varepsilon_{x}(\mathrm{~mm} \mathrm{mrad})$ & 0.2 & 0.3 & 0.3 & 0.4 \\
\hline$\sigma_{E} / E(\%)$ & 0.02 & 0.02 & 0.03 & 0.02 \\
$N_{u}$ & 1100 & 1670 & 2500 & 2220 \\
$L_{u}(\mathrm{~m})$ & 20.0 & 30.0 & 45.0 & 40.0 \\
Net gain $G$ & 1.64 & 1.63 & 1.29 & 1.23 \\
\hline Spectral FWHM (meV) & 14.7 & 18.4 & 16.2 & 15.3 \\
Temporal FWHM (fs) & 170 & 148 & 156 & 165 \\
$P_{\text {peak }}(\mathrm{MW})$ & 8.4 & 6.1 & 0.64 & 1.1 \\
Photons/pulse & $8.7 \times 10^{8} 5.5 \times 10^{8} 6.0 \times 10^{7} 1.2 \times 10^{8}$ \\
\hline \hline
\end{tabular}

assuming near backscattering from the crystal surfaces. The major drawback to this scheme is that, because the x-ray energy is set by Bragg's law $E=E_{H} / \cos \Theta$, it cannot be easily varied for $\Theta \ll 1$. In order to vary the $\mathrm{x}$-ray energy, one can use the four-mirror geometry shown in Fig. 4. For this configuration, the x-ray angle of incidence $\Theta$ is adjusted at all four mirrors so as to vary the photon energy while maintaining the same round-trip length. As previously mentioned, the basic physics of this cavity are similar to that of the simple resonator cavity discussed in Sec. III C, but the additional elements will also decrease the round-trip reflectivity, while the finite angular acceptance of the crystals at $\Theta \geqslant 1^{\circ}$ will introduce additional physics constraints. However, our GINGER simulations do not include the angular response of the Bragg crystals. For the present study, we assume that the focusing elements produce a $0.25 \mu \mathrm{rad} \mathrm{x}$-ray divergence on the Bragg crystals, a value much less than the crystal acceptance of $\sim 1-4 \mu \mathrm{rad}$. Thus, while we expect that the results presented here should give a reasonable indication of the four-mirror requirements and x-ray properties, definitive predictions will have to be deferred until further code modifications have been made to more completely model the $2 \mathrm{D}$ physics of this geometry.

We list the preliminary simulation parameters and results for several possible four-mirror x-ray FEL oscillator configurations in Table III. The basic required parameters and output characteristics are quite similar to those listed in Table I, although the electron beam current (and, hence, charge) has been increased to 20 A to overcome the additional losses. Additionally, we include two separate results for each of the 9- and 14-keV photon energies: one for which we use the theoretical crystal reflectivities and assume a $5 \%$ loss for each of two focusing elements, and 
TABLE III. Possible undulator, beam, and optical cavity parameters for the 4-mirror, tunable FEL. For all cases the electron beam energy $E_{\text {beam }}=7 \mathrm{GeV}$, current $I_{\text {beam }}=20 \mathrm{~A}$, energy spread $\sigma_{\mathrm{E}}=1.4 \mathrm{MeV}$, the rms beam length $\sigma_{t}=1 \mathrm{ps,} \mathrm{while} N_{u}=3000$ and the undulator gap is $5 \mathrm{~mm}$. The lower box contains radiation characteristics of the $\mathrm{x}$-ray pulses that are couple through the thin mirror assuming $4 \%$ transmission, and include the "idealized" reflectivities on the left and the more conservative ones on the right, as described in the text.

\begin{tabular}{|c|c|c|c|c|c|}
\hline Parameter & \multicolumn{2}{|c|}{$9.131 \mathrm{keV}$} & \multicolumn{2}{|c|}{$14.4125 \mathrm{keV}$} & $20.514 \mathrm{keV}$ \\
\hline$\lambda_{u}(\mathrm{~cm})$ & \multicolumn{2}{|c|}{1.76} & \multicolumn{2}{|c|}{1.66} & 1.50 \\
\hline$N_{u}$ & \multicolumn{2}{|c|}{3000} & \multicolumn{2}{|c|}{3000} & 3000 \\
\hline FEL $K$ & \multicolumn{2}{|c|}{1.52} & \multicolumn{2}{|c|}{1.32} & 1.05 \\
\hline$\varepsilon_{x, n}(\mathrm{~mm} \mathrm{mrad})$ & \multicolumn{2}{|c|}{0.2} & \multicolumn{2}{|c|}{0.2} & 0.1 \\
\hline Linear $G_{0}$ & \multicolumn{2}{|c|}{0.86} & \multicolumn{2}{|c|}{0.58} & 0.56 \\
\hline Bragg crystal & \multicolumn{2}{|c|}{$\mathrm{C}\left(\begin{array}{lll}3 & 3 & 3\end{array}\right)$} & \multicolumn{2}{|c|}{$\mathrm{C}\left(\begin{array}{lll}3 & 3 & 7\end{array}\right)$} & C(3 3311$)$ \\
\hline Tuning range & \multicolumn{2}{|c|}{$6.2 \%$} & \multicolumn{2}{|c|}{$6.0 \%$} & $3.5 \%$ \\
\hline & $R_{\mathrm{tot}}=0.81$ & $R_{\mathrm{tot}}=0.72$ & $R_{\mathrm{tot}}=0.83$ & $R_{\mathrm{tot}}=0.73$ & $R_{\mathrm{tot}}=0.83$ \\
\hline$P_{\text {peak }}(\mathrm{MW})$ & 3.9 & 1.8 & 2.0 & 0.9 & 1.6 \\
\hline Spectral FWHM (meV) & 1.57 & 1.78 & 1.78 & 2.06 & 1.25 \\
\hline$\sigma_{\tau} \sigma_{\omega}(\mathrm{rms})$ & 1.05 & 0.93 & 1.39 & 1.13 & 0.71 \\
\hline Photons/pulse & $4.6 \times 10^{9}$ & $2.0 \times 10^{9}$ & $1.2 \times 10^{9}$ & $0.4 \times 10^{9}$ & $0.92 \times 10^{9}$ \\
\hline
\end{tabular}

another that includes an additional $10 \%$ loss. This decreases the peak power and photon flux by approximately a factor of 2, but still provides coherent, nearly Fourierlimited x-ray pulses with FWHM bandwidths between 1 and $2 \mathrm{meV}$. Simulation results indicate that there is sufficient gain to tune the photon energy by about $\pm 3 \%$ for the lower two energies. At $20 \mathrm{keV}$, a tuning in energy of $\pm 1.7 \%$ can be achieved. The pulse profiles in time and spectra look similar to those of the two-crystal cavity shown in Fig. 8.

We might also consider using the compressed, $100 \mathrm{~A}$ beam option presented in Sec. IVB, with the hope of increasing the linear gain and thereby decreasing the beam emittance or cavity reflectivity requirements. This attractive possibility cannot be addressed without including the angular divergence of the Bragg crystal because the $\mathrm{x}$-ray spectrum produced by a 100 fs electron beam will fill the entire spectral bandpass, and the radiation will be affected by the full $R(\lambda, \Theta)$. We plan to investigate these physics issues in subsequent work, in which angledependent reflectivity will be included in our simulation model. In this case, however, each individual Bragg crystal breaks the assumed axial symmetry, treating positive and negative angles with respect to the optical axis differently. Nevertheless, the combined acceptance of all four crystals and their respective drift spaces is approximately symmetric in angles, and the total transformation between the focusing optics $M_{1}$ and $M_{2}$ in Figs. 4 and 6 can be expressed in the angular representation of the field as a simple multiplication that is axially symmetric. For this reason, GINGER can adequately represent the cavity in Figs. 4 and 6. Since this optical configuration can be used to collimate the beam outside the undulator and decrease the effect of the finite angular crystal acceptance, we believe this to be the most attractive tunable oscillator, but numerically assessing other geometries may require a fully three-dimensional field representation.

\section{CONCLUSIONS}

In this paper we investigated the performance of the $\mathrm{x}$-ray FEL oscillator using two-dimensional GINGER simulations. These simulations solved the standard FEL equations in the undulator, and included diffraction, focusing, and the full Bragg crystal reflectivity in the cavity propagation. We confirm that the primary effect of the crystal is to filter the radiation in frequency and to delay it in time. We also find that the penetration of the $\mathrm{x}$ rays into the crystals results in an effective delay that requires compensation via a cavity length reduction of tens of microns. The essential conclusions $[4,24]$ based on 1D arguments and idealized crystal reflectivities have been confirmed: a highquality $\left(\varepsilon_{x}=0.2 \mathrm{~mm} \mathrm{mrad}\right)$, low-charge $(25-50 \mathrm{pC})$ electron beam of 1 ps duration can produce $\mathrm{x}$ rays with 1-10 MW of peak power in an extremely narrow, nearly Fourier-limited bandwidth $\sim 1-3 \mathrm{meV}$ FWHM. Additionally, compressing the electron beam by a factor of 10 increases the single-pass gain, and one can consider easing the requirements on the electron beam emittance, the cavity reflectivity, and/or the undulator length. We have shown that with a $100 \mathrm{~A}, \varepsilon_{x}=0.3 \mathrm{~mm}$ mrad beam, one can overcome $50 \%$ losses using $30 \mathrm{~m}$ of undulator to produce $\sim 5 \times 10^{8}$ photons in a 150 fs FWHM pulse whose bandwidth is $18 \mathrm{meV}$ (FWHM). Furthermore, one might easily imagine electron and undulator parameters anywhere between these two cases, although compressing the electron beam further will result in shorter $\mathrm{x}$-ray pulses only if suitable crystal planes with a broader spectral bandwidth are chosen. Finally, the four-mirror geometry 
appears to be an attractive way of making this source tunable in photon energy by $3 \%-6 \%$, although to make definitive statements in this regard will require including the angular dependence of the crystal reflectivity into the FEL simulation code.

\section{ACKNOWLEDGMENTS}

This work was supported by U.S. Department of Energy, Office of Basic Energy Sciences, Contract No. DE-AC02$06 \mathrm{CH} 11357$.

[1] P. Emma for the LCLS Team, in Proceedings of the 23rd Particle Accelerator Conference, Vancouver, Canada, 2009 (IEEE, Piscataway, NJ, 2009).

[2] sPring-8 Compact Self-Amplified Spontaneous Emission Source Conceptual Design Report, http://www-xfel .spring8.or.jp.

[3] http://xfel.eu/.

[4] K.-J. Kim, Y. Shvyd'ko, and S. Reiche, Phys. Rev. Lett. 100, 244802 (2008).

[5] R. Colella and A. Luccio, Opt. Commun. 50, 41 (1984).

[6] W. M. Fawley, Lawrence Berkeley Laboratory Technical Report No. LBNL-49625.

[7] Y. Shvyd'ko, X-Ray Optics-High Energy-Resolution Applications (Springer, New York, 2004).

[8] Z. Huang and R. D. Ruth, Phys. Rev. Lett. 96, 144801 (2006).
[9] G. Dattoli, A. Marino, A. Renieri, and F. Romanelli, IEEE J. Quantum Electron. 17, 1371 (1981).

[10] P. Elleaume, IEEE J. Quantum Electron. 21, 1012 (1985).

[11] K.-J. Kim and Y. V. Shvyd'ko, Phys. Rev. ST Accel. Beams 12, 030703 (2009).

[12] A. E. Siegman, Lasers (University Science Books, New York, 1986).

[13] K.-J. Kim, in Proceedings of the 1987 Particle Accelerator Conference (IEEE, Washington, DC, 1987).

[14] K.-J. Kim, Nucl. Instrum. Methods Phys. Res., Sect. A 246, 71 (1986).

[15] E. D. Courant and H. S. Snyder, Ann. Phys. (Leipzig) 3, 1 (1958).

[16] H. Wiedemann, Particle Accelerator Physics (SpringerVerlag, Berlin-Heidelberg, 1993).

[17] K.-J. Kim, Nucl. Instrum. Methods Phys. Res., Sect. A 318, 489 (1992).

[18] D.-M. Smilgies, Appl. Opt. 47, E106 (2008).

[19] C. Ferrero, D.-M. Smilgies, C. Riekel, G. Gatta, and P. Daly, Appl. Opt. 47, E116 (2008).

[20] S. J. Hahn, W. M. Fawley, K.-J. Kim, and J. A. Edighoffer, Nucl. Instrum. Methods Phys. Res., Sect. A 358, 167 (1995).

[21] W. H. Southwell, Opt. Lett. 3, 100 (1978).

[22] Y. V. Shvyd'ko, S. Stoupin, A. Cunsolo, A. H. Said, and X. Huang, Nature Phys. 6, 196 (2010).

[23] S. Stoupin and Y. V. Shvyd'ko, Phys. Rev. Lett. 104, 085901 (2010).

[24] R. R. Lindberg and K.-J. Kim, Phys. Rev. ST Accel. Beams 12, 070702 (2009). 